\section{Réduire les pertes d'azote dans l'élevage}

\section{Peyraud J.-L., Cellier P., Donnars C., Vertès F. (coord.) et al., 2014, Ed. Quae, 166 p.}

Résumé - Le livre "Réduire les pertes d'azote dans l'élevage » s'attache à identifier les moyens de lutter efficacement contre les risques de pollution de l'environnement par les composés azotés réactifs dus à cette production. Les formes d'azote réactif sont décrites, leurs sources identifiées, leurs flux mesurés. Les moyens de réduire ces flux et les politiques à conduire pour y parvenir sont analysés.

Ce livre est le produit, par un groupe d'experts qualifiés, d'une expertise scientifique collective ${ }^{1}$ demandée à l'Inra en 2012 par les ministères en charge de l'Agriculture et de l'Écologie.

Dans leur introduction, les auteurs (au nombre de 28) rappellent que le développement de l'élevage à l'échelle mondiale fait l'objet de débats, notamment en raison des risques de pollution de l'environnement qu'il entraîne. L'Europe s'est saisie du problème, avec des résultats contrastés. Le cycle de l'azote est esquissé à grands traits, la profonde évolution amenée par l'industrie des engrais à partir du début $\mathrm{du} 20^{\mathrm{e}}$ siècle soulignée. Puis les auteurs définissent le périmètre de l'expertise, centrée sur l'exploitation d'élevage en France, et rappellent les grandes lignes de la méthode utilisée par l'INRA pour ce type de travail.

Le corps du texte est construit en trois parties.

La première, intitulée : "Contribution de l'élevage à la pression azotée sur les territoires », comprend elle-même trois chapitres.

L'azote est présent dans la nature sous différentes formes, dont l'azote de l'air, le plus abondant, mais non réactif, ainsi que sous des formes réactives, susceptibles de se combiner à d'autres éléments. L'apparition de ces formes, leurs transformations, leurs cheminements et leurs flux, constituent la « cascade de l'azote ». Un même élément, par exemple l'ion nitrate, peut être tour à tour un élément indispensable à la vie ou un polluant, quand il s'accumule dans les nappes d'eau souterraines.

Les effets de l'agriculture et plus particulièrement de l'élevage sur cette cascade sont particulièrement importants, avec des conséquences préoccupantes sur l'environnement. Ils

\footnotetext{
${ }^{1}$ « Les flux d'azote liés aux élevages, réduire les pertes, rétablir les équilibres ».
}

sont l'objet de discussions dans les instances internationales, européennes et françaises et sont examinés à différentes échelles : depuis l'échelle mondiale, par le transfert d'aliments du bétail par exemple, jusqu'à des entités beaucoup plus petites, les bassins de collecte d'eaux de boisson, les exploitations, les cultures, les prairies permanentes, les bâtiments. Les auteurs de l'étude expliquent également comment on en est arrivé à la situation actuelle, évoquant les raisons historiques, économiques et de politique de protection de l'environnement. Les cas français, caractérisés par des régions très contrastées en matière d'élevage, et européen sont privilégiés, ce qui n'exclut pas quelques comparaisons avec d'autres cas si nécessaire (Amérique du Nord et du Sud, notamment). La faible efficacité des mesures déjà prises en France pour limiter les effets de l'azote sur l'environnement est relevée.

La partie 2, « Description et quantification des flux d'azote dans les élevages », vise à décrire plus en détail la cascade et à quantifier les flux, aux différentes échelles déjà citées. Les méthodes et outils pour quantifier ces flux sont présentés dans leur diversité, leurs intérêts et limites discutés d'un point de vue aussi bien théorique que pratique, comme outils d'aide à la décision pour réduire les pollutions. Ce sont notamment les bilans en azote à l'échelle de l'exploitation, ou de l'un des ateliers gérés par l'exploitant ou les calculs d'efficience de l'azote, rapport entre les quantités d'azote présentes dans les produits (animaux, végétaux) et les quantités mobilisées pour les produire. Les principaux facteurs limitant les actions à partir de ces indicateurs, autres que les problèmes éventuellement rencontrés pour les calculer, sont d'une part la diversité des situations et la difficulté à trouver des solutions adaptées à chacune, d'autre part, la nécessité d'avoir l'accord des acteurs concernés pour traiter les problèmes à une échelle supérieure à celle de l'exploitation d'élevage. De nombreuses questions restent en suspens, faute d'outils adaptés pour les traiter, ou n'ont pu être abordées que par modélisation. Des techniques pour réduire les effets de l'azote sur l'environnement sont présentées, par exemple pour conserver et traiter les déjections, pour la valorisation agronomique des effluents, soit au niveau des exploitations qui les produisent, soit au niveau d'un ensemble d'exploitations. Ces solutions doivent être soigneusement analysées, car un gain concernant un maillon de la chaîne de production peut se traduire par une fuite lourde de conséquences au niveau du maillon suivant. L'intérêt de systèmes de production constitués par l'agriculture à bas niveau d'intrants ou l'agriculture biologique est souligné. Quelques exemples d'aménagements à l'échelle d'un territoire, co-financés par des sociétés ou des collectivités (villes, pour leur approvisionnement en eau potable), sont également décrits. 
La troisième partie porte plus spécifiquement sur « Utiliser moins et mieux l'azote »; cette fois-ci en considérant dans son ensemble la chaîne :

« Pratiques $^{2}>$ Émissions $>$ Changements d'état $>$ Exposition > Impacts »

et en utilisant les méthodes et outils décrits en deuxième partie, de façon à identifier les plus efficaces en fonction des résultats recherchés. Puis les auteurs abordent les instruments de régulation des politiques pour réduire les pollutions azotées et en discutent l'efficacité : ainsi sont passés en revue les divers types d'incitations financières, taxes, subventions assorties ou non de sanctions, les réglementations, les instruments associant quota et pénalités, les possibilités d'appliquer le principe pollueur-payeur. La préoccupation de réduire les risques qui pèsent sur l'environnement n'est pas récente. Mais les dispositions prises pour les réduire n'ont pas, du moins jusqu'à présent, permis d'obtenir les résultats escomptés. Quelques pistes sont explorées pour améliorer la situation. À l'échelle des exploitations, ou des ateliers qui la constituent, des progrès sont possibles pour améliorer les bilans, accroître l'efficience de l'azote, tout en préservant le revenu des agriculteurs. Mais il n'y a pas encore en France d'outil pour étudier dans sa globalité le fonctionnement d'une filière et il paraît actuellement difficile d'agir sur certaines des causes des pollutions comme la concentration des activités de production animale dans certaines régions, liée à la dissociation entre agriculture et élevage.

Cette partie se termine par l'analyse d'évènements qui pourraient affecter la gestion de l'azote : évolution des prix de l'énergie, poursuite de la déréglementation des marchés agricoles, agrandissement des exploitations, évolution de la consommation de produits animaux.
En conclusion, les auteurs rappellent le rôle majeur de l'élevage dans les flux d'azote : en France, près des trois-quarts des apports en azote des fertilisants aux cultures sont utilisés pour la production d'aliments du bétail, qui consomme $70 \% \mathrm{de}$ la biomasse produite et qui, lui-même, produit 1820000 tonnes d'effluents. Les auteurs résument l'état des connaissances en soulignant que les efforts ont longtemps porté sur le nitrate essentiellement, mais que les travaux sur l'ensemble de la cascade de l'azote, plus récents, doivent être accrus. Ils reviennent ensuite sur les pistes qu'il faut continuer à développer : les démarches d'optimisation des pratiques, de concertation concernant les territoires denses en animaux ou particulièrement vulnérables, le traitement des effluents en vue de leur exportation des zones où l'élevage est très concentré, enfin la déconcentration dans ces zones. Les outils à la disposition des autorités nationales seraient à réexaminer. Des besoins en recherche résultent de ces travaux, pour une meilleure connaissance de la dynamique de l'azote dans les systèmes de production, mais également en matière d'efficacité des politiques publiques.

Cet ouvrage est riche en figures et tableaux de données récentes.

En tête de chacune des grandes parties, un bref rappel de ce qui a déjà été présenté et un résumé de ce qui va l'être en facilite la lecture. Il contient également une bibliographie abondante.

Il intéressera aussi bien un public spécialisé, producteurs, décideurs, mais aussi enseignants, chercheurs et étudiants, qu'un public large, sensibilisé à ces questions et soucieux de bien comprendre les enjeux et les difficultés à traiter les problèmes de pollution de l'environnement liés à l'élevage.

Didier Picard (didier.picard@grignon.inra.fr)

\footnotetext{
${ }^{2}$ Il s'agit des pratiques culturales ou d'élevage des exploitants agricoles, mais aussi de celles de tous les acteurs intervenant dans la chaîne.
} 